\title{
Evaluation of Steric and Substituent Effects in Phenols by Competitive Reactions of Dimethyl Ether Ions in a Quadrupole Ion Trap
}

\author{
Gerald F. Bauerle, Jr. and Jennifer S. Brodbelt \\ Department of Chemistry and Biochemistry, University of Texas at Austin, Austin, Texas, USA
}

\begin{abstract}
Competitive reactions of dimethyl ether ions are used to probe the steric and substituent effects of substituted phenols and anisoles in a quadrupole ion trap. The relative percentages of protonation and methylene substitution from the reactions of dimethyl ether ions show a correlation with size, location, and number of substituents on the aromatic ring. Although gas-phase basicity measurements of the phenols show no discernible correlation with the percentages of competitive reactions, semiempirical calculations show a good correlation between the trend in heats of formation and the trend in methylene substitution percentage. Reactions with deuterated compounds show that the methylene substitution reaction occurs on the ring. (J Am Soc Mass Spectrom 1995, 6, 627-633)
\end{abstract}

$\mathrm{S}$ teric and substituent effects have been studied extensively in solution and have been shown to have marked effects upon the outcome, mechanisms, and kinetics of reactions [1]. In the gas phase, however, few systematic studies have been reported that show these effects on ion-molecule reactions. Steric effects are known to affect gas-phase proton transfer [2-7]. Most aromatic systems show a decrease in the rate of proton transfer with increasing size of substituents near the site of protonation, especially in the ortho position [5]. Even highly basic compounds may demonstrate low proton transfer rates if the protonation site is sufficiently blocked [6]. Exceptions to the decrease in the rate of proton transfer have been noticed where the transition state for proton transfer involves a perpendicular, rather than a coplanar, configuration to the ring [7]. A decrease in the efficiency of product formation from ionic acetylation of substituted phenols as the size of substituents at the ortho positions increases has been seen [8]. The effect was not noted for meta substituted phenols. Another study showed an increasing extent of fragmentation with increasing bulk of a substituent at the para position of a substituted 2,6-tert-butylphenol during methane chemical ionization [9].

Because of our ongoing interest in characterization of structural factors that affect the reactivity of molecules in the gas phase, we have undertaken a systematic study of substituent effects by comparing the relative extent of three competitive reactions for a series of substituted aromatic compounds. The probe

Address reprint requests to Professor Jennifer S. Brodbelt, Department of Chemistry and Biochemistry, University of Texas at Austin, Welch Hall 1.202, Austin, TX 78712-1167. reactant ions, generated from dimethyl ether, undergo proton transfer, methylene substitution, and methylation [10] with aromatic compounds. It has been shown that protonation of phenol by isobutane as well as methylation of phenol by methyl fluoride and methyl chloride both occur on the ring [11]. With anisole, methylene substitution also occurs on the ring [10]. With the reactive sites located on the ring, the competition between these reactions has allowed us to correlate the relative abundances of the three types of product ions formed with the substituent effects of the substrates.

As described herein, the relative extent of protonation versus methylene substitution varies with the number, position, and nature of alkyl substituents on the phenol ring. Two effects - steric effects and activating effect - of the alkyl groups have been evaluated. In addition, deuterated compounds, semiempirical molecular orbital calculations, and collisionally activated dissociation were used to study dissociation mechanisms of the product ions and to gain further insight into the reactions.

\section{Experimental}

All experiments were performed in a Finnigan (Finnigan-MAT, San Jose, CA) quadrupole ion trap that used the mass-selective instability mode for ion detection [12]. Dimethyl ether was used as the reagent gas for chemical ionization of the phenols and related compounds. The dimethyl ether was ionized by electron impact, and the desired ions were isolated by using the apex isolation mode [13]. The ratio of $\mathrm{m} / \mathrm{z} 45$ to $m / z 47$ ions was maintained at $4: 1$ at the beginning of each reaction. The ions were then allowed to react with 
the analyte for $25 \mathrm{~ms}$. Fifty scans were averaged for each spectrum. The reported results were obtained from an average of at least three trials conducted on different days. Each analyte was introduced through a heated leak valve to an uncorrected pressure of $1 \times$ $10^{-6}$ torr. Dimethyl ether and helium were introduced through needle valves at nominal pressures of $1 \times 10^{-5}$ and $8 \times 10^{-5}$ torr, respectively. The manifold temperature was held at $110^{\circ} \mathrm{C}$ for all experiments. Collisionally activated dissociation (CAD) of the products was accomplished by isolation of the desired product ion and its activation by application of an ac voltage across the endcap electrodes at a $q$ value between 0.3 and 0.5 . The time of activation was $5 \mathrm{~ms}$ with tickle voltages between 450 and $600 \mathrm{mV}$.

Relative gas-phase basicities were obtained by application of the ligand exchange method [14]. Protonated dimethyl ether was isolated and allowed to react with two neutral, aromatic substrates. One protonated substrate was isolated and was allowed to react with the other neutral substrate. This process was reversed by isolating the other protonated substrate and allowing it to undergo proton transfer. The substrate with the higher gas-phase basicity will retain the proton.

Semiempirical calculations were performed by using MOPAC 6.0 [15] on a DEC 4000 VLC (Digital Equipment Corp., Maynard, MA). Numerous chemically reasonable structures were minimized by using the AM1 hamiltonian [16], and the minimum energy conformer for each structure is reported. Heats of formation were rounded to the nearest kilocalorie per mole.

Dimethyl ether was obtained from MG Industries (Valley Forge, PA). Perdeuterated dimethyl ether was obtained from Isotec (Miamisburg, $\mathrm{OH}$ ). The remainder of the compounds were obtained from Aldrich Chemical Co. (Milwaukee, WI), and all chemicals were used as received.

\section{Ion-Molecule Reactions with Dimethyl Ether Ions}

Upon electron impact and self-ion-molecule reactions, dimethyl ether forms two primary ions: one at $m / z 45$ $\left(\mathrm{CH}_{2}=\mathrm{OCH}_{3}\right)^{+}$and the other at $m / z \quad 47$ $\left(\mathrm{CH}_{3} \mathrm{OCH}_{3}\right) \mathrm{H}^{+}$[10]. The $m / z 47$ ion is an excellent protonating agent, and the $m / z 45$ ion reacts with aromatic substrates to give either the methylene substitution product $[M+13]^{+}$or the methylated product $[\mathrm{M}+15]^{+}$, dependent on the nature of the substrate [10]. In general, those aromatic compounds that have activating substituents will form the $[M+13]^{+}$ion, whereas those with deactivating substituents will form the $[M+15]^{+}$ion [10].

In these experiments, the reactions of the two types of dimethyl ether ions with aromatic substrates occur simultaneously and competitively in a kinetic sense. Because each of the two competing reactions is pseudo first order, the ratio of product ions at a well-defined fixed time of $25 \mathrm{~ms}$ can be used to represent a slice of the rate constant curves. The reaction with a lower activation barrier will occur faster and irreversibly and leads to a more abundant product. The activation barriers are largely dependent on the specific structure of the aromatic substrate and, therefore, the relative abundances of the product ions can be correlated with the reaction efficiencies. The result is competing reactions that provide a sensitive probe of structural factors that influence the gas-phase reactivities of the phenol-anisole substrates. A series of phenols and anisoles was chosen for this study (Figure 1). Various alkyl-substituted phenols and analogous anisoles were used to vary the nature and position of the substituents. Compounds were chosen to block reactions at certain sites or to restrict reactions in an area of the aromatic ring.

Product percentages from the reaction of dimethyl ether ions with these compounds are given in Figures $2-4$. Trends can be seen in the relative percentages of $[M+1]^{+}$and $[M+13]^{+}$products. The formation of $[\mathrm{M}+15]^{+}$is an insignificant process for all of the compounds and will not be discussed further. As illustrated in Figure 2, the methylene substitution percentage initially increases across the series and then decreases. In a similar trend, there is an initial decrease in the percentage of the protonation process, followed by an increase. Apparently, the alkyl groups specifically activate the ring for methylene substitution. As the number of alkyl groups increases, a steric effect that blocks the methylene substitution reaction starts to dominate and then the protonation reaction becomes the prevalent reaction.

Possible reasons for these trends are derived from the proposed mechanisms for these reactions (Scheme I). For phenol, the protonation percentage is greater than that of methylene substitution, which indicates that the proton transfer is the faster reaction. The methylene substitution reaction has an intermediate rearrangement that consists of a proton migration that slows the reaction. When methyl groups are added to

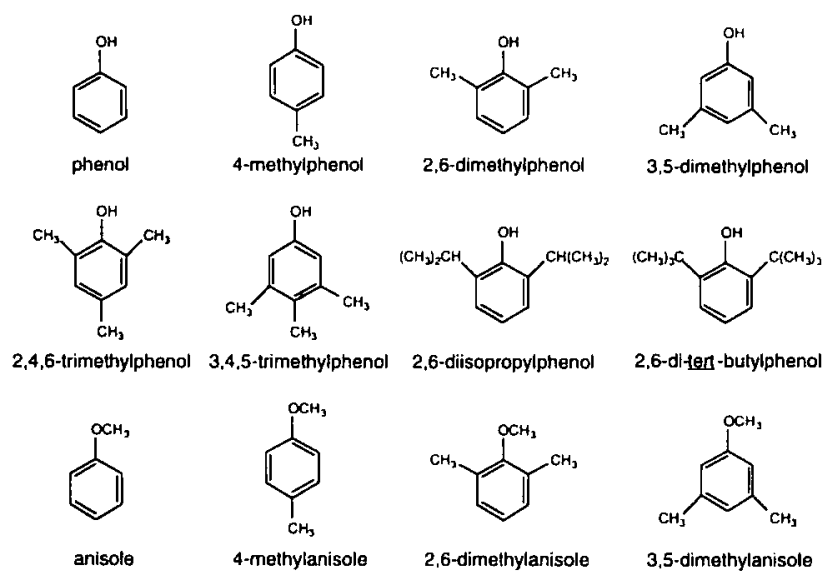

Figure 1. Compounds used for ion-molecule reactions with dimethyl ether ions. 


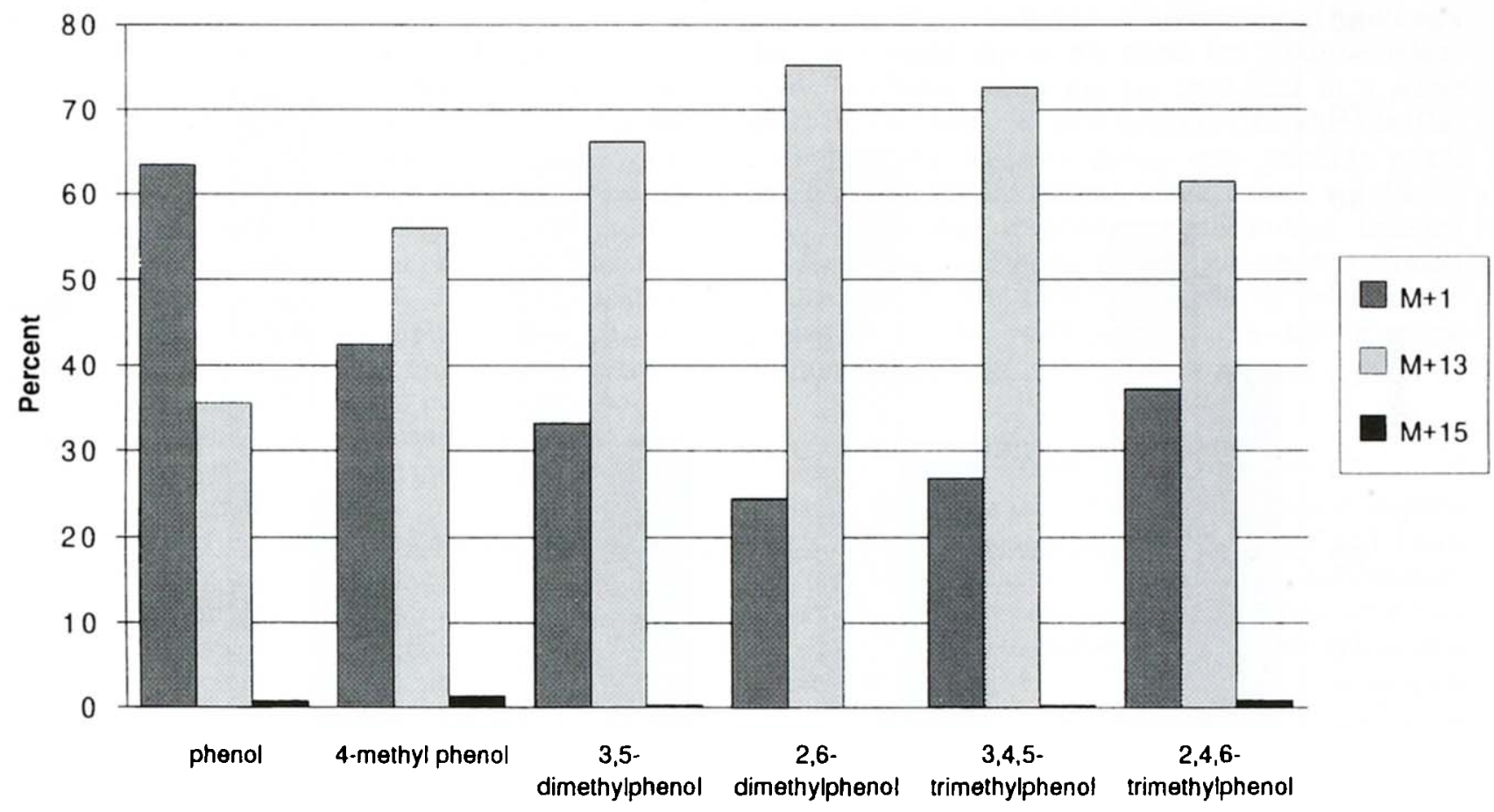

Figure 2. Relative percentages of products from the reaction of dimethyl ether ions. The percentages are reproducible to $\pm 5 \%$.

the ring, electron density increases on the phenol ring, which makes it more nucleophilic. Both $\left(\mathrm{CH}_{3} \mathrm{OCH}_{2}^{+}\right)$ and $\left(\mathrm{CH}_{3} \mathrm{OCH}_{3}\right) \mathrm{H}^{+}$act as electrophiles in the reactions, but the added electron density on the aromatic ring apparently stabilizes the intermediate for methylene substitution more efficiently and enhances the methanol elimination and formation of $[\mathrm{M}+13]^{+}$rela- tive to the proton transfer process. Overall, as the number of methyl groups increases, the rate of the methylene substitution process increases faster relative to the protonation process. This effect causes the difference in relative percentages of the two competing reactions to become larger up to the point that three methyl groups are added to the ring. At this point, a

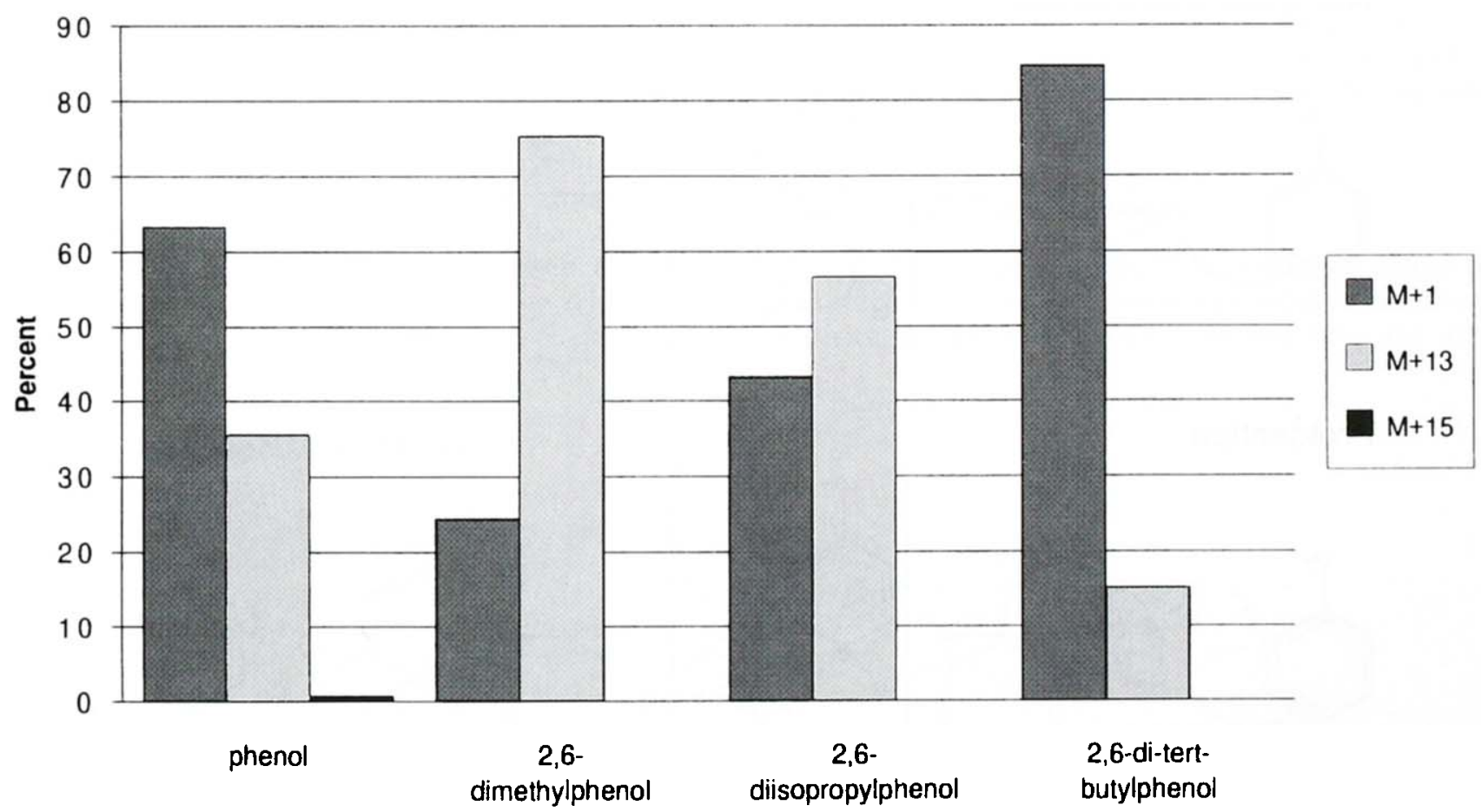

Figure 3. Relative percentages of products from the reaction of dimethyl ether ions. The percentages are reproducible to $\pm 5 \%$. 


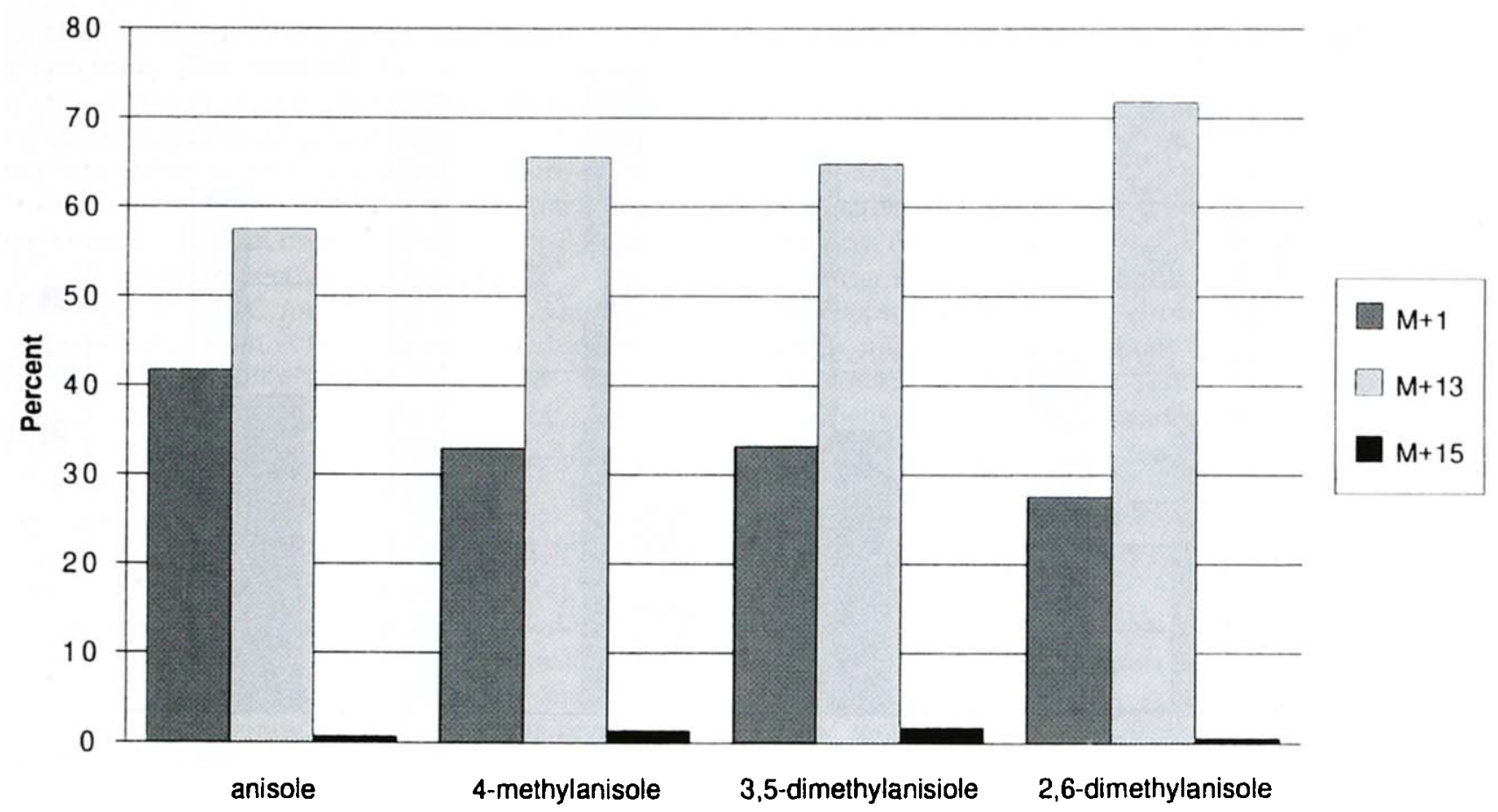

Figure 4. Relative percentages of products from the reaction of dimethyl ether ions. The percentages are reproducible to $\pm 5 \%$.

steric effect from the methyl groups significantly interferes with the formation of the intermediate for methylene substitution. The proton-bound complex is less susceptible to the steric effects of the methyl groups and therefore an increase in the relative percentage of protonation is observed.
In Figure 2, several points can be inferred about the positional selectivity of methylene substitution. The percentage of methylene substitution for 3,5-dimethylphenol, where all ring sites are ortho or para to all substituents, is less than that of 2,6-dimethylphenol, where the ring sites are meta to at least one sub-

\section{Methylene Substitution}

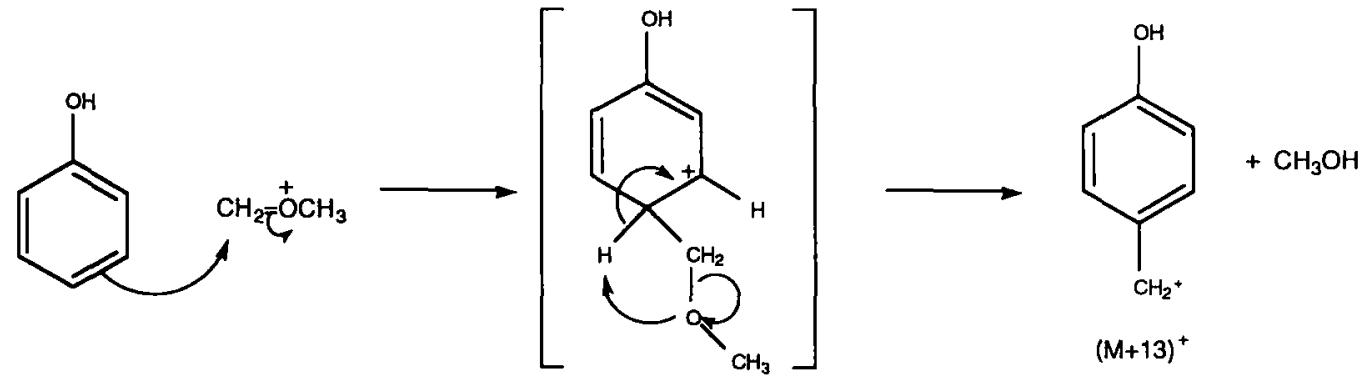

\section{Protonation}
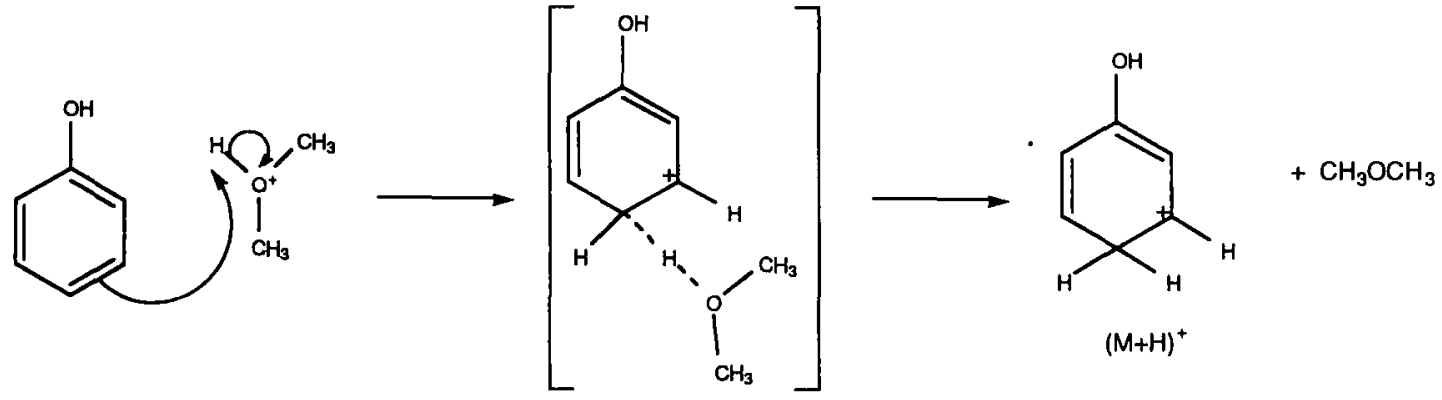

$(\mathrm{M}+\mathrm{H})^{+}$

Scheme I. Proposed mechanisms for the methylene substitution and protonation of phenol by dimethyl ether ions. 
stituent. If steric effects are considered, it can be seen that 2,6-dimethylphenol has three adjacent ring carbons that lack substituents, whereas 3,5-dimethylphenol has no adjacent carbons without substituents. Therefore, methylene substitution should be favored for 2,6-dimethylphenol, as verified in Figure 2 by a $9 \%$ difference in the product of the methylene substitution reaction for the two dimethylphenol isomers. The same logic can be used to explain why methylene substitution is greater for 3,4,5-trimethylphenol than 2,4,6-trimethylphenol. Although there are no ring carbons without neighboring substituents in either compound, 3,4,5-trimethylphenol has two unsubstituted ring positions adjacent to the hydroxy group. The hydroxy group is smaller than a methyl group and therefore has a diminished steric effect. Because both of the unsubstituted ring positions in the 2,4,6-trimethylphenol have neighboring methyl groups, methylene substitution is more sterically hindered and thus shows a relative abundance that is $11 \%$ smaller than that of 3,4,5-trimethylphenol. In addition, the activated ortho and para positions are all blocked in 2,4,6-trimethylphenol, which should further decrease the extent of the methylene substitution process. Semiempirical calculations were employed to give a better understanding of site selectivity and will be discussed later.

Figure 3 illustrates the trend related to the addition of increasingly bulky groups to the phenol ring. The initial activation effect of the added methyl groups causes the methylene substitution percentage to increase. As the size of the substituents at the ortho position increases, the steric effect becomes larger, which causes a decrease in the methylene substitution percentage. For 2,6-di-tert-butylphenol, this steric blocking results in almost exclusive protonation.

Figure 4 shows the corresponding trends for a series of anisoles. The methylene substitution percentage is substantially greater than the protonation percentage for anisole, the reverse of the case observed for phenol. This result occurs because a methoxy group can donate more electron density to the ring than a hydroxy group, thereby activating the ring more effectively for the methylene substitution process. The relative percentages of the methylene substitution product in- creases for the substituted anisoles as well, although there is a slight dip in the trend for 3,5-dimethylanisole. The latter result can be attributed to a steric effect that is similar to that noted for the phenols. The methyl on the methoxy group may partially block attack at the ortho positions, which causes a decrease in the percentage of methylene substitution. Because the percentages for the methylene substitution process of the anisoles are greater or equal to those of the phenols, it can be inferred that the location of attack also is on the ring.

\section{Site of Methylene Substitution}

To confirm the site of methylene substitution, experiments that used phenol-(ring- $d_{5}$ ) were performed. From the proposed mechanism of methylene substitution (Scheme I), it can be seen that the result of an attack on the ring will be the addition of $\mathrm{CH}_{2}$ with the loss of a deuterium to give $[\mathrm{M}+12]^{+}$. An attack on the oxygen would give the addition of $\mathrm{CH}_{2}$ with the loss of a hydrogen atom to give $[M+13]^{+}$. The results of the experiment showed exclusive substitution on the ring, leading to $[M+12]^{+}$, with no detectable substitution on the oxygen. Therefore, the methylene substitution process occurs on the ring.

To further affirm the proposed methylene substitution mechanism, CAD experiments were performed. Upon activation, the $[M+13]^{+}$ion of phenol shows losses of 28 and $30 u$ to give ions at $m / z 79$ and 77 , respectively [15]. If the $m / z 79$ ion is isolated and activated, it gives the $m / z 77$ ion as a product, which indicates a two-step reaction from the original $[M+$ $13]^{+}$ion to the deprotonated benzene ion may be operative. A possible mechanism for the loss of $\mathrm{CO}$ is given in Scheme 2. This mechanism indicates an [M+ $13]^{+}$product structure that stems from methylene substitution at the ring.

\section{Gas-Phase Basicities}

To gain further insight into the competitive nature of the reactions of dimethyl ether ions, relative gas-phase basicity measurements were performed for the aro-

\section{Dissociation of $(M+13)+$}

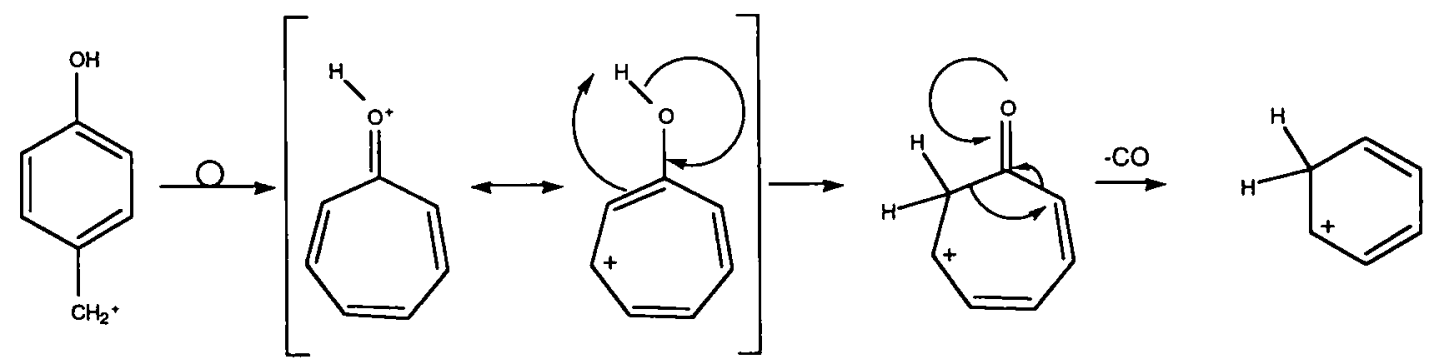

Scheme II. Proposed mechanism for the loss of $\mathrm{CO}$ from the $[\mathrm{M}+13]^{+}$methylene substitution product of phenol. 


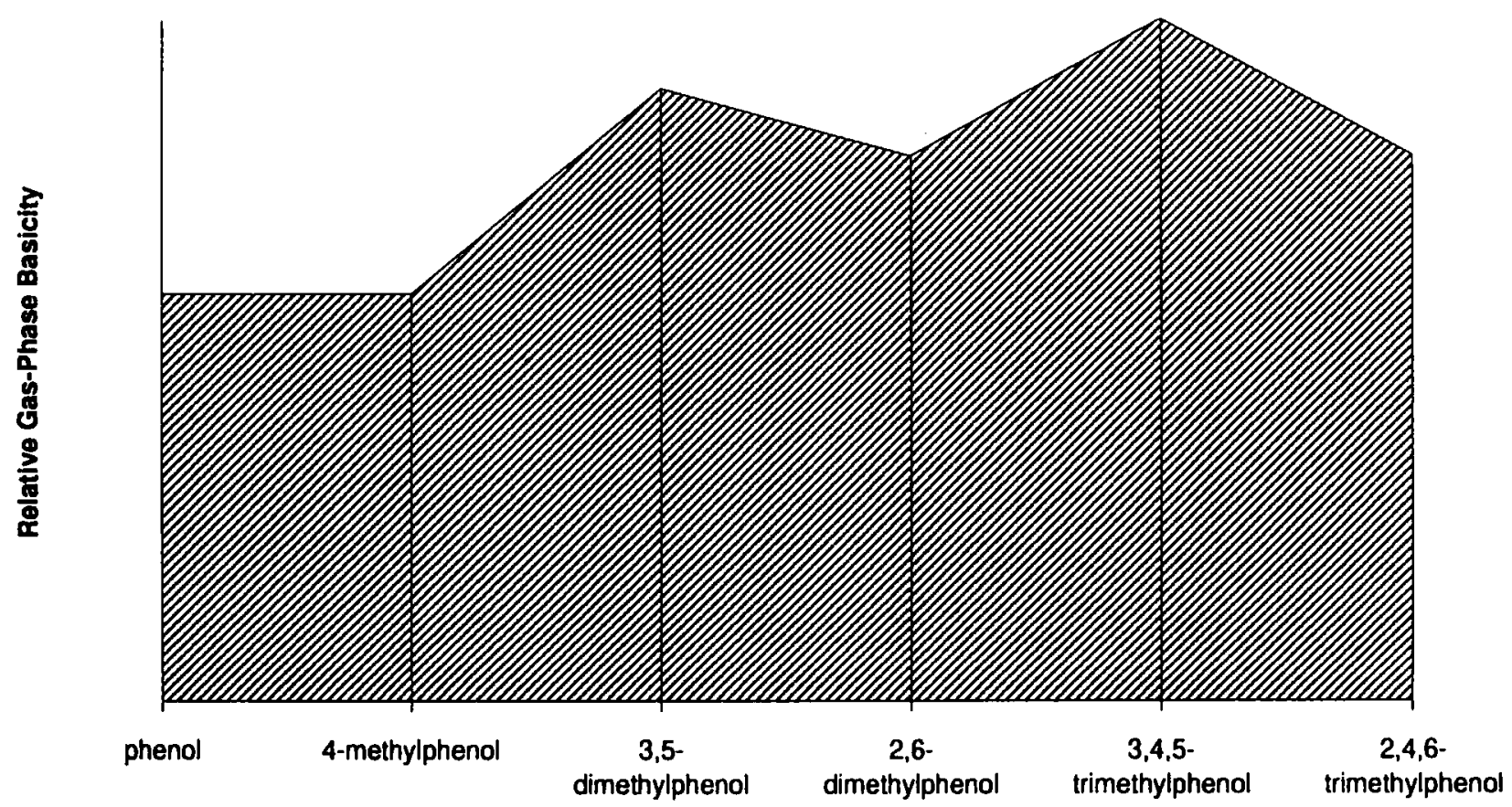

Figure 5. Relative gas-phase basicities of the phenols in Figure 2. Arbitrary units are used.

matic compounds. Gas-phase basicity is one measure of a molecule's ability to bind a proton. In the absence of steric blocking, a higher basicity of a compound should correspond to faster proton-transfer reactions that involve the dimethyl ether reactant ions, which causes a larger percentage of protonation compared to methylene substitution. The relative basicities, as measured by ligand exchange, are shown in Figure 5 . The gas-phase basicity generally increases from left to right across the graph in accordance with work done on substituted pyridines [18]. This trend does not directly track the trend seen for the relative extent of protonation in Figure 2. Likewise, there is no direct correlation between the extent of methylene substitution and gasphase basicity. The gas-phase basicity - a thermochemical parameter-does not reflect or predict the trends in the competitive reactions of the dimethyl ether ions. This result further supports a "kinetic-based" rationalization for the observed trends of the competing reactions. Steric and substituent effects of each aromatic substrate, rather than the stabilities of the product ions, play the more important roles that influence the reaction efficiencies.

\section{Semiempirical Molecular Orbital Calculations for Methylene Substitution Products}

The semiempirical results from the compounds were used to help determine site selectivity of the methylene substitution reaction. The heats of formations for the various products of the methylene substitution reaction are given in Table 1 . For phenol, the most stable products are those where substitution occurs at the ortho and para positions, which corresponds well with the favored products from solution chemistry [19]. The results also show attack on the oxygen to be highly unfavorable in relation to attack at the other positions on the ring, in agreement with experimental work. For the other compounds, the most stable products are those in which methylene substitution involves the ortho or para position relative to the meta position.

\section{Conclusions}

The competitive reactions of dimethyl ether ions are a viable method to probe the effects of substituents on the reactivities of phenols and anisoles. The relative percentages of products from these ions are sensitive to location, number, and size of the substituents on the aromatic ring. Methylene substitution shows a greater response to activating effects of the substituents, whereas protonation is less affected by steric effects

Table 1. Computational results from the semiempirical molecular orbital calculations for heats of formation of $[M+13]^{+}$products (in kilocalories per mole)

\begin{tabular}{lcccc}
\hline & \multicolumn{5}{c}{ Site of Attack } \\
\cline { 2 - 5 } \multicolumn{1}{c}{ Compound } & Oxygen & Ortho & Meta & Para \\
\hline \hline Phenol & 193 & 171 & 181 & 170 \\
4-Methylphenol & 184 & 162 & 171 & - \\
3,5-Dimethylphenol & 176 & 150 & - & 152 \\
2,6-Dimethylphenol & 177 & - & 161 & 154 \\
3,4,5-Trimethylphenol & 169 & 145 & - & - \\
2,4,6-Trimethylphenol & 168 & - & 159 & - \\
\hline
\end{tabular}


from the substituents. The gas-phase basicity does not correlate with the efficiency of the two competing reactions. Semiempirical calculations also have shown that the most stable products are those where the methylene substitution occurs on the ortho or para position. Results from the chemical ionization reactions of nondeuterated and deuterated compounds show that the methylene substitution reaction likely occurs on the ring.

The results from this study indicate the possibility to use this type of competitive reactions in other systems, such as aromatics that have substituents other than alkyl groups. Also, use of the methylation reaction as a probe for aromatic systems with deactivating substituents is a possibility.

\section{Acknowledgments}

This work was supported by ACS-PRF (25485-AC5), NIH (R01GM46723), NSF (CHE 9122699 and CHE 9357422), the Welch Foundation (F1155), the Dreyfus Foundation, and the Sloan Foundation.

\section{References}

1. Isaacs, N. S. Physical Organic Chemistry; Longman Group: Essex, England, 1987, pp 135-144, 282-329.

2. Jasinski, J. M.; Brauman, J. I. J. Am. Chem. Soc. 1980, 102, 2906-2913.

3. Meot-Ner (Mautner), M; Sieck, L. W. I. Am. Chem. Soc. 1983, 105, 2956-2961.
4. Meot-Ner (Mautner), M; Smith, S. C. J. Am. Chem. Soc. 1991, 113, 862-869.

5. Attinà, M.; Cacace, F. Int. J. Mass Spectrom. Ion Processes 1992, 109, 95-104.

6. Houriet, R.; Rolli, E. New I. Chem. 1987, 11, 221-224.

7. Büker, H.; Grützmacher, H. Int. J. Mass Spectrom. Ion Pracesses 1991, 109, 95-104.

8. Benezra, S. A.; Bursey, M. M. J. Am. Chem. Soc. 1972, 94, 1024.

9. Kadentsev, V. I.; Krokhin, A. V.; Chizov, O. S.; Ershov, V. V. Izv. Akad. Nauk SSSR, Ser. Khim. 1978, 11, 2532-2538.

10. Brodbelt, J. S.; Liou, C.; Donovan, T. Anal. Chem. 1991, 63, 1205-1209.

11. Wood, K. V.; Burinsky, D. J.; Cameron, D.; Cooks, R. G. J. Org. Chem. 1983, 48, 5236-5242.

12. Stafford, G. C., Jr.; Kelley, P. E.; Syka, J. E. P.; Reynolds, W. E.; Todd, J. F. J. Int. I. Mass Spectrom. Ion Processes 1984, 60, 85-98.

13. Weber-Brabau, M.; Kelley, P. E.; Syka, J. E. P.; Bradshaw, S. C.; Brodbelt, J. S. Proceedings of the 35th Annual Conference of the American Society for Mass Spectrometry, 1987; p 1114.

14. Brauman, J. I.; Blair, L. K. J. Am. Chem. Soc. 1970, 92, 5986-5992.

15. Stewart, J. P. Quantum Chemistry Program Exchange 455, Indiana University, Bloomington, IN.

16. Dewar, M. J. S.; Zoebisch, E. G.; Healy, E. F.; Stewart, J. J. P. J. Am. Chem. Soc. 1985, 107, 3902-3909.

17. Donovan, T.; Liou, C.; Brodbelt, J. J. Am. Soc. Mass Spectrom. 1992, 3, 39-46.

18. Hopkins, H., Jr.; Jahagirdar, D.; Moulik, P.; Aue, D.; Webb, H.; Davidson, W.; Pedley, M. J. Am. Chem. Soc. 1984, 106, 4341-4348.

19. Solomons, T. W. G. Organic Chemistry; John Wiley: New York, 1988; pp 648-663. 